\title{
$35 \mathrm{ku}$ 的 PF18-3 分子在小鼠胸腺细胞凋亡 亚群上的特异表达
}

\section{王宏 李 燕 庞学文 陈慰峰“}

(北京医科大学免疫学系, 卫生部医学免疫学重点实验室, 北京 100083。* 联系人)

摘要 PF18-3 抗体是一株大鼠抗小鼠胸腺基质细胞单克隆抗体. 在以往的共培养研究中发现 该抗体可抑制小鼠胸腺树突状细胞系( MTSC4) 诱导的胸腺细胞调亡作用. 目的是观察 PF18-3 抗体识别分子(PF18-3 分子) 的特点及其在 MTSC4 诱导的胸腺细胞调亡中的作用. 利用流式 细胞仪对 PF18-3 分子的表达进行了特性分析. 发现PF18-3 分子除表达于 MTSC4 表面外. 还表 达于 Con A 活化的胸腺细胞上, 但新鲜胸腺细胞表面无表达. 胸腺细胞与 MTSC4 共育 $48 \mathrm{~h}$ 后, PF18-3 分子表达上调. 流式细胞仪分析 PI 染色检测调亡相关亚二倍体及 PF18-3 抗体染 色的结果表明, PF18-3 分子特异表达于高亚二倍体含量的调亡胸腺细胞. 经免疫共沉淀和 Western blotting 鉴定, 调亡胸腺细胞表达的 PF18-3 分子的分子量为 $35 \mathrm{ku}$. 该分子可能参与胸 腺细胞调亡过程.

\section{关键词 胸腺细胞 细胞凋亡 单克隆抗体 介导分子}

胸腺阴性选择过程涉及自身反应性胸腺细胞调亡 ${ }^{11]}$. 更多未经选择的胸腺细胞由于 TCR 缺陷不能行使功能, 不能被活化, 而经历无选择死亡(death by neglect) 的调亡过程 ${ }^{2]}$, 但何种 信号分子引发胸腺内发育中 $\mathrm{T}$ 细胞发生程序性死亡仍不清楚. 研究参与上述选择过程的膜表 面或胞内分子, 对了解 $\mathrm{T}$ 细胞的发育及中枢耐受的形成具有重要意义.

PF18-3 抗体为我室自建的抗小鼠胸腺基质细胞 (Mouse Thymic Stromal Cell, MTSC) 单克隆 抗体, 研究曾发现该抗体可明显抑制小鼠树突状细胞系(MTSC4) 对胸腺细胞调亡的促进作 用 ${ }^{[3]}$, 提示 PF18-3 抗体识别分子(简称 PF18-3 分子) 可能参与 MTSC4 诱导的胸腺细胞调亡过 程. 本文进一步分析了 PF18-3 分子的调亡相关作用.

\section{1 材料与方法}

（ ）细胞与培养基. MTSC1 和 MTSC4 分别为本室建立小鼠胸腺髓质型上皮细胞系及树 突状细胞系, 胸腺细胞取自 4 周龄 Balb/c 雌性小鼠. DMEM + $10 \%$ 新生牛血清 (NCS) 培养基 常规培养.

（ ）胸腺细胞的 Con A 活化. 胸腺细胞以 $6 \times 10^{5} / \mathrm{mL}$ 的浓度接种, 培养基中加入 Con A 至终浓度 $3 \mu \mathrm{g} / \mathrm{mL}$, 取不同时间活化细胞进行分析.

（）MTSC 与胸腺细胞的共育. MTSC1 及 MTSC4 在培养瓶中培养至 $5 \times 10^{5} / \mathrm{mL}$ 浓度, 将 10 倍浓度新鲜胸腺细胞加入共育, 不同时间将胸腺细胞从 MTSC 细胞表面轻轻吹打下来, 进行表型分析.

（ ）细胞表型分析. $1 \times 10^{6}$ 细胞分别加入 1: 100 稀释的苂光素标记抗体直接染色, 或 经间接荧光抗体染色, 即以 1: 1000 稀释的腹水来源的大鼠抗小鼠胸腺基质细胞抗体; $4{ }^{\circ} \mathrm{C} 45$ $\mathrm{min}$; 以含 $2 \%$ 小牛血清的等张平衡盐缓冲液 (BSS+ $2 \% \mathrm{NCS}$ ) 洗涤 2 次后, 再加入苂光素标记 
羊抗大鼠 $\operatorname{IgG}$ 抗体, $4{ }^{\circ} \mathrm{C} 45 \mathrm{~min}$; 以 BSS $+2 \% \mathrm{NCS}$ 洗涤 2 次后, 加入 FACS 保存液( $1 \%$ 甲醛, $2 \%$ 葡萄糖, $0.1 \% \mathrm{NaN}_{3}$, 溶于 PBS)，以流式细胞仪(FACScan, BD 公司) 分析细胞表型. 采用门 技术将细胞分成不同亚群, 对不同亚群细胞表型进行分析, Lysis 毛统计软件分析数据.

（）FACS 检测调亡细胞的亚二倍体 DNA. 细胞加入 $75 \%$ 乙醇过夜, 次日用 PBS 洗 2 次, 加入 PI (碘化丙啶) 低渗染液 ( $50 \mathrm{\mu g} / \mathrm{mL}$ PI, $0.1 \%$ 柠檬酸钠, $0.1 \%$ Triton X-100), 以 FACS 检测细胞核 DNA 中亚二倍体的调亡 DNA 波峰.

（ ) 细胞膜蛋白的提取. $5 \times 10^{7}$ 细胞以 PBS+ $2 \% \mathrm{NCS}$ 洗 2 次, 加入 $1 \mathrm{~mL}$ 三去污剂裂解 液 $(50 \mathrm{mmol} / \mathrm{L}$ Tris $\mathrm{HCl}(\mathrm{pH}=8.0), 150 \mathrm{mmol} / \mathrm{L} \mathrm{NaCl}, 0.02 \%$ 叠氮钠, $0.1 \% \mathrm{SDS}, 1 \%$ Nonidet $\mathrm{P}-40$ (NP-40)，0.5\%去氧胆酸钠), $1 \mu \mathrm{L} 10 \mathrm{mg} / \mathrm{mL}$ 的 Aprotinin , $1 \mu \mathrm{L} 10 \mathrm{mg} / \mathrm{mL}$ 的 Leupeptin, 2 忙 $1.74 \mathrm{mg} / \mathrm{mL}$ 的 PMSF, 冰浴 $1 \mathrm{~h}, 7000 \mathrm{r} / \mathrm{min}$ 离心 $10 \mathrm{~min}$, 上清冻存, 用前以 $8000 \mathrm{r} / \mathrm{min}$, 离 心 $10 \mathrm{~min}$, 弃沉淀.

( ) 免疫共沉淀. 将经偶联缓冲液 $(0.1 \mathrm{~mol} \mathrm{NaHCO} / 3 / 0.5 \mathrm{~mol} \mathrm{NaCl})$ 透析过夜的腹水抗 体按说明书偶联至 CNBr 活化的 Sepharose CL-4B(Pharmacia 公司), 按文献[1] 方法进行免疫共 沉淀.

（）SDS-PAGE 及银染. 共沉淀蛋白进行 $12 \%$ 还原性 SDS-PAGE 电泳，电泳后将胶以 $30 \%$ 乙醇/ $10 \%$ 冰醋酸固定过夜, 再浸入 $30 \%$ 乙醇/ $0.4 \mathrm{~mol} / \mathrm{L} \mathrm{NaAc/} 0.5 \%$ 戊二酫/ $0.1 \%$ 硫代 硫酸钠 $(\mathrm{pH}=6.0)$ 中, $50{ }^{\circ} \mathrm{C} 8 \mathrm{~min}$, 再经 $30 \%$ 乙醇洗 2 次, 去离子水洗 3 次, 将胶置于 $40{ }^{\circ} \mathrm{C}$ $0.1 \% \mathrm{AgNO}_{3}$ (含 $50 \mu \mathrm{L}$ 甲醛 $/ 100 \mathrm{~mL}$ ) 中, 室温避光染色 $30 \mathrm{~min}$, 再经水洗 3 次后, 浸于 $2.5 \%$ $\mathrm{NaHCO}_{3}$ (含 $40 \mu \mathrm{L}$ 甲醛 $/ \mathrm{mL}$ ) 溶液中显色 $2 \sim 5 \mathrm{~min}$, 以 $3 \%$ 冰醋酸终止反应.

( ) Western blotting. SDS-PAGE 电泳后. 将蛋白从胶中恒压 $15 \mathrm{~V}$ 电转移过夜至硝酸纤 维膜上, 封闭液 (磷酸盐缓冲液 $(\mathrm{PBS})+5 \%$ 脱脂奶粉) 中室温捊育 $1 \mathrm{~h}$, 含 1: 800 PF 18-3 腹水抗 体的杂交液中室温孵育 $2 \mathrm{~h}$, 以 PBS/ $0.1 \%$ Tween-20 洗 3 次, 将膜继续与含 $1: 1000 \mathrm{HRP}$ 标记 的羊抗大鼠 $\mathrm{k} / \lambda$ 轻链 (Sigma 公司) 的杂交液中室温捊育 1 . $\mathrm{h}$, 以 $\mathrm{PBS} / 0.1 \%$ Tween-20 洗 3 次后, 将 ECL 试剂盒显色 底物液(Amersham 公司) 等体积混合, 滴加于膜上 $1 \mathrm{~min}$, 压 $\mathrm{X}$ 光片，适宜时间后显影定影.

\section{2 结果}

\subsection{Con A 活化胸腺细胞 PF18-3 分子的动态表达}

新鲜分离的小鼠活的胸腺细胞不表达 PF18-3 分子, 经 Con A 活化后, 开始表达该分子, 并随培养时间延长, 该分子表达增加. 对照抗体 RS21-C6 同时进行 FACS 分 析, 未见其识别分子在胸腺细胞表面表达, 延长 Con A 刺激与培养时间也不能诱导 RS21-C6 抗体识别分子的表 达上调 (图 1), 说明 PF18-3 分子是与其识别抗体特异结 合的结果，而非抗体的非特异吸附.

2.2 PF18-3 在调亡胸腺细胞亚群中的特异表达

利用门技术将 Con A 活化的胸腺细胞分为 2 个亚群

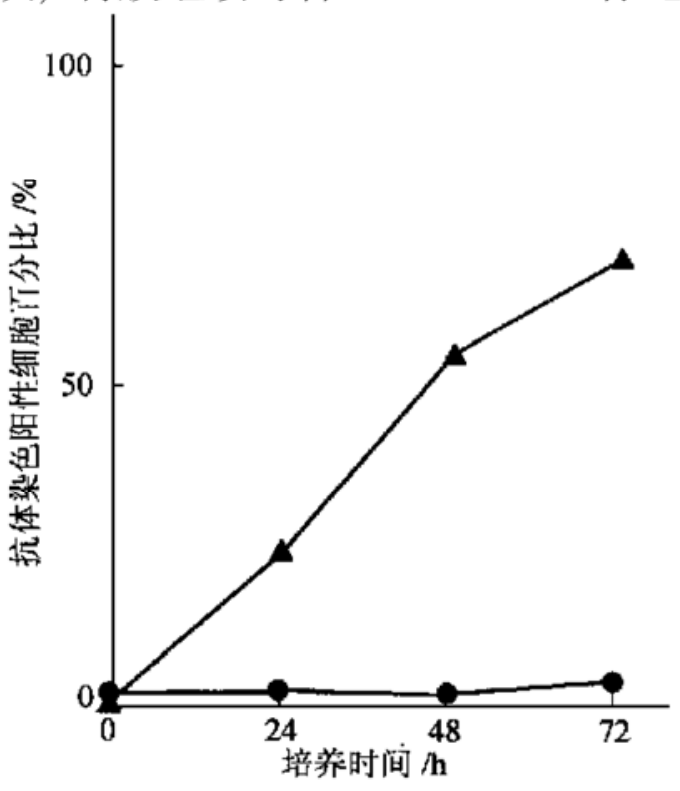

图 1 Gon A 活化胸腺细胞 PF18-3 分子表达动态 -示对照抗体, \示 PF18-3 抗体 
$\mathrm{R} 2$ 和 R3(见图 2), R3 为多颗粒小细胞亚 群, R2 为大细胞亚群. 同时对这两个细胞 亚群进行 PF18-3 抗体染色和亚二倍体 PI 200染色分析(图 3)，可见 PF18-3 分子几乎全漹 部表达于 R3 亚群，而该亚群又恰是富含亚 二倍体的细胞亚群. 鉴于亚二倍体是调亡 细胞的重要检测指标, 上述结果表明 PF183 分子特异表达于调亡胸腺细胞亚群.

2.3 胸腺基质细胞作用对 PF18-3 分子表 \& 达的影响

图 4 可见胸腺细胞与胸腺基质细胞共 育 $24 \mathrm{~h}$ 后其 PF18-3 分子表达水平与单独胸

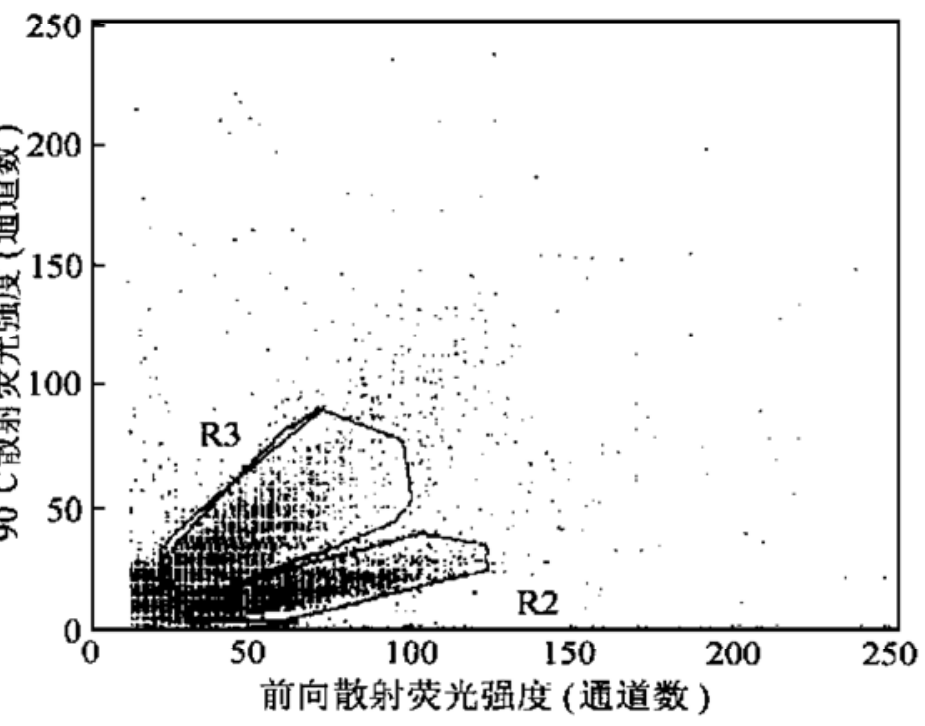
腺细胞培养比较无显著改变. 当共育时间 延长至 $48 \mathrm{~h}$, 共育后胸腺细胞的 PF18-3 分

图 2 FACS 门技术对 ConA 活化胸腺细胞进行亚群分类 子表达水平明显高于胸腺细胞单独培养对照, 提示 PF18-3 分子可能参与介导胸腺基质细胞 诱导胸腺细胞发生调亡的作用.

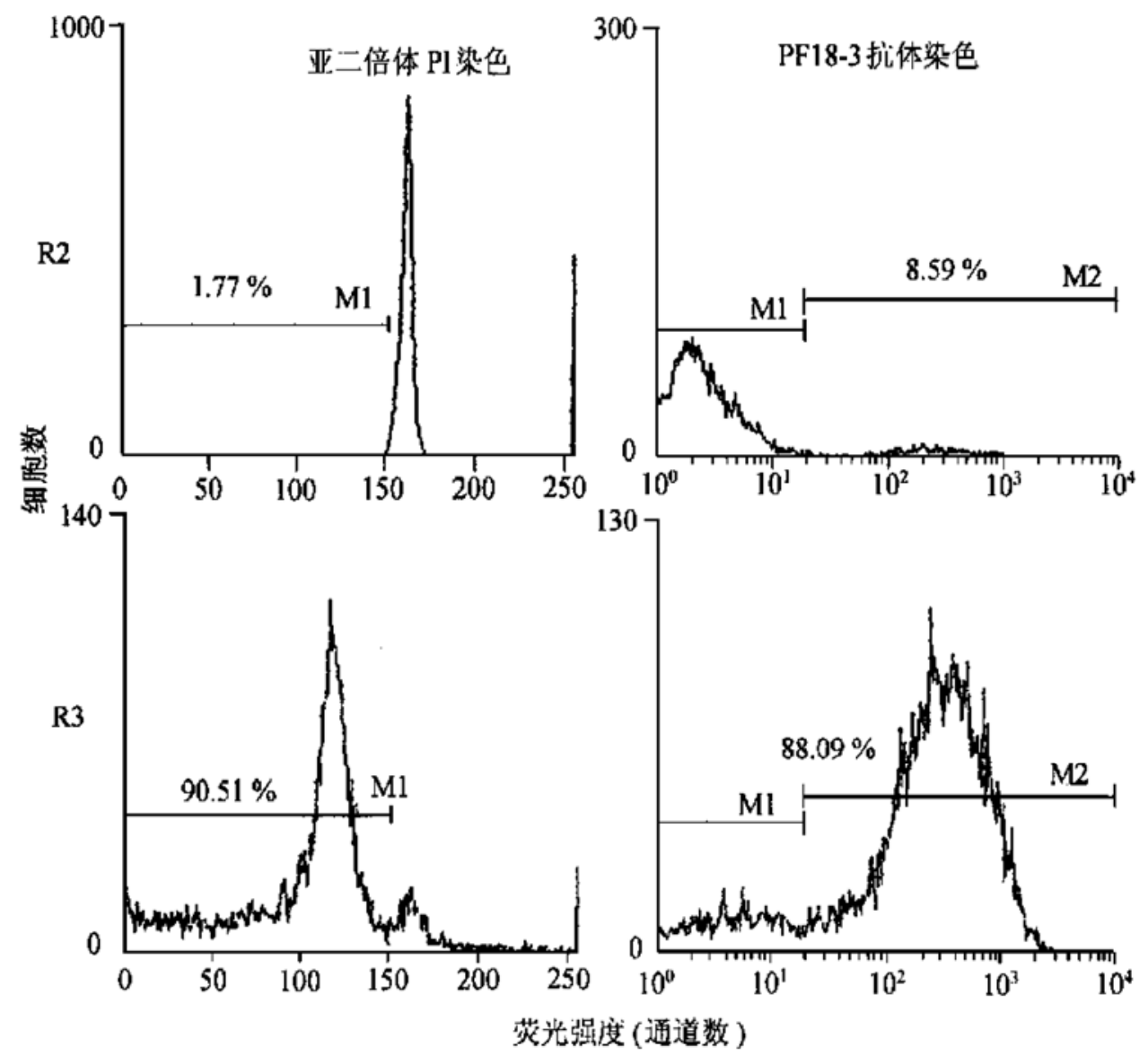

图 3 PF18-3 分子在胸腺细胞调亡亚群中的表达

2.4 PF18-3 分子的分子量及抗体识别特异性鉴定

提取 Con A 活化 $48 \mathrm{~h}$ 的胸腺细胞膜蛋白, 经 PF18-3 抗体免疫共沉淀后行 SDS-PAGE 电 
泳, 胶经银染后在 $35 \mathrm{ku}$ 处可见明显的沉淀带, 在相同区域各抗体及细胞对照均无类似沉淀带 产生, 说明此沉淀为 PF18-3 抗体特异免疫共沉 淀产物(图 5).

上述共沉淀产物经 SDS-PAGE 电泳后电转移 至硝酸纤维膜上，用 PF18-3 抗体行蛋白免疫印 迹杂交, 图 6 可见 $35 \mathrm{ku}$ 处出现特异性杂交带, 其带型与银染带型一致，说明该沉淀带为 PF183 抗体特异性识别分子.

\section{3 讨论}

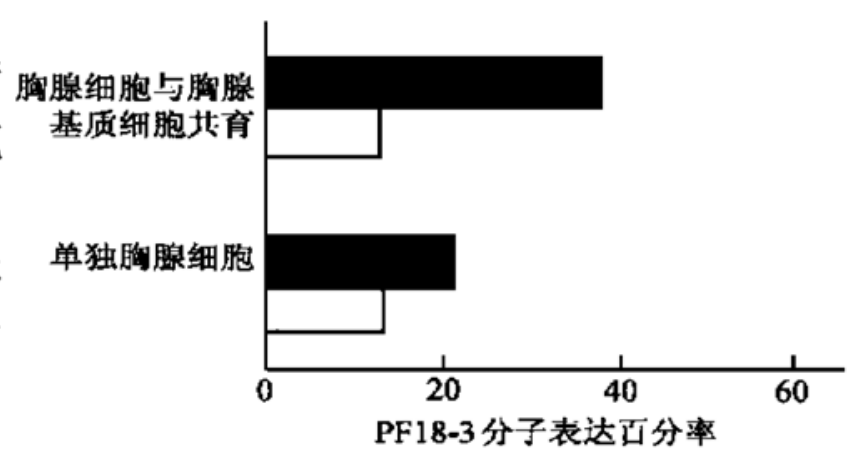

$\mathrm{T}$ 细胞在胸腺内发育过程中通过与胸腺微

图 4 胸腺基质细胞对胸腺细胞 PF18-3

分子表达的作用

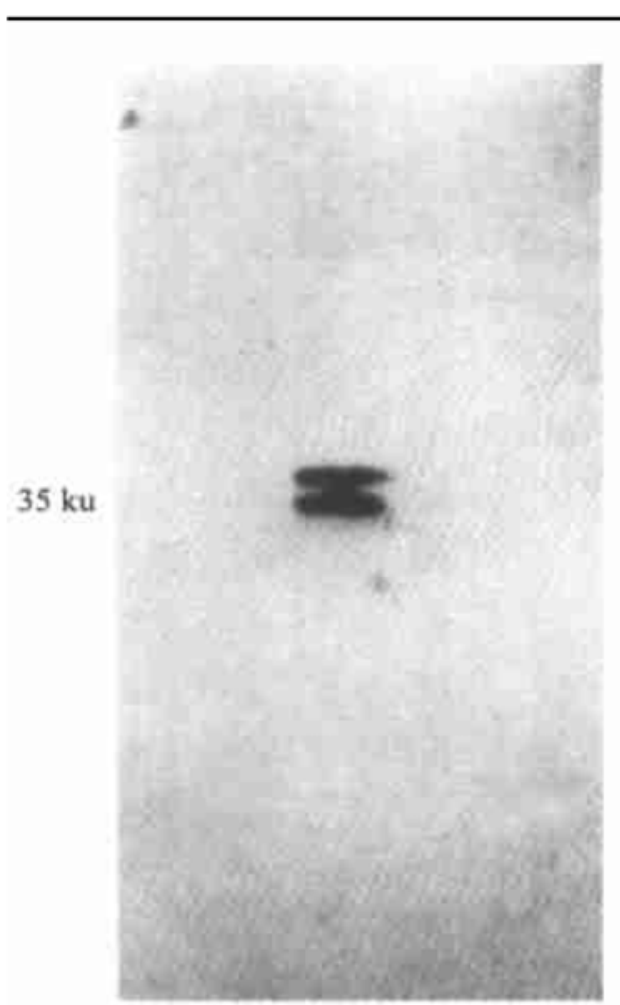

图 5 PF18-3 分子的免疫共沉淀分析 M 一蛋白标准, A-PF18-3 抗体对照, B-PF18_3 共沉 淀, C-RS21-C6 抗体对照, D-RS21-C6 共沉淀, E胸腺基质细胞对照

环境相互作用获得不同信号，阳性选择信号使细胞得以存活并分化发育为成熟 $\mathrm{T}$ 细胞，输出 胸腺执行免疫功能，阴性选择信号或无信号将引发胸腺细胞的调亡过程. 为寻找参与上述过 程的细胞表面分子，我们用本室建立的多种抗 MTSC 单抗进行研究，发现其中的 PF18-3 mAb 具有抑制胸腺树突状细胞诱导胸腺细胞发生调亡的作用，提示其识别分子可能参与胸腺细胞 发育中的调亡介导作用. 由于胸腺内 $\mathrm{T}$ 细胞发育过程中的调亡介导分子还不清楚，经典 FasFasL (配基) 通路可能在胸腺内不起主要作用 ${ }^{[4]}$, 即存在有未知调亡介导分子的可能，为此本 文对 PF18-3 分子的特性及其与调亡的关系进行了研究. 
以往对 PF18-3 抗体特性的研究曾发现, 该抗体在胸腺基质细胞和胸腺细胞染色均为阳 性, 但新鲜胸腺细胞需经固定后才能呈染色阳性，说明该抗体识别分子为 MTSC 和胸腺细胞 的共有抗原分子，但未经刺激的胸腺细胞该分子存在于胞浆中而非细胞膜表面 ${ }^{[5]}$. 本研究发 现胸腺细胞经 Con A 活化后可在细胞膜表面表达 PF18-3 分子，随活化时间延长，该分子的表 达持续增加. 此高表达状态为研究此分子的性质提供良好的分析细胞模型. 对此活化细胞进 行 FACS 染色分析, 可见其细胞散点图呈左上和右下 2 个亚群分布. 利用门技术对这两个细 胞亚群进行 PF18-3 分子表达和调亡特征性亚二倍体分析，发现左上细胞亚群为高亚二倍体的 调亡胸腺细胞亚群，而 PF18-3 分子又正是高表达于此亚群细胞，说明该分子确实与细胞调亡 相关，联系其相应抗体对调亡的抑制作用，不能排除其介导胸腺细胞调亡的可能性. 考虑胸 腺基质细胞对胸腺细胞发育及调亡的重要作用，我们利用本室所建胸腺基质细胞系与胸腺细 胞共育, $48 \mathrm{~h}$ 后亦发现胸腺细胞 PF18-3 分子表达上调, 提示该分子可能参与导致胸腺细胞调 亡的胸腺基质细胞-胸腺细胞间相互作用过程.

免疫共沉淀及 Western blotting 结果证明活化胸腺细胞表面 PF18-3 分子为分子量 $35 \mathrm{ku}$ 左 右蛋白, 免疫共沉淀产物经 SDS-PAGE 电泳银染后显示为两条位置相近的沉淀带. 而此分子 在胸腺基质细胞表面则表现为 $31 \mathrm{ku}$ 的单一带 ${ }^{[6]}$. 这种不同细胞间分子量的差异及同一细胞 上两种相近分子量蛋白的存在提示此蛋白糖基化的可能. 比较该蛋白与调亡相关分子的关 系，Fas 及 Fas 配基的分子量与该蛋白不一致，Fas 与该蛋白的表达动态也不相同. 近来发现 在细胞凋亡过程中某些通常在胞浆中或浆膜层存在的分子会移位至细胞表面，如热休克蛋 白 $^{[7]}$ 及磷脂 ${ }^{[8]}$. PF18-3 分子在胸腺细胞凋亡过程中也有类似的移位表达现象, 但其分子量远 小于热休克蛋白，另文研究中亦发现 PF18-3 分子在细胞调亡过程中的表达动态及分布与磷脂

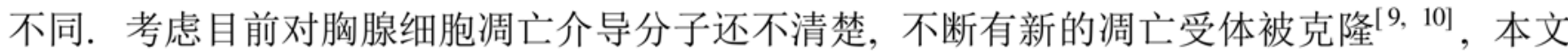
涉及的 PF18-3 分子存在作为新型调亡相关分子的可能性. 对该分子的基因克隆将有助于确 认这种可能性，并对进一步探讨胸腺细胞阴性选择过程的机制具有重要意义.

致谢 本工作为国家“八六三”计划(批准号：863-102-11-01-01) 和卫生部科研基金( 批准号：96-1-258) 资助项 目.

\section{参 考 文 献}

1 Nossal G J V. Negative selection of lymphocytes. Cell, 1994, 76: 229 239

2 Surh C D, Sprent J. T- cell apoptosis detected in situ during positive and negative selection in the thymus. Nature, 1994, 372: 100 103

3 Dong Haidong, Chen Jing, ChenWeifeng. Membrane molecules in induction of apoptosis of thymocytes by mouse thymic dendritic cells which express Fas ligands. Science in China, 1998, 41(2): 189 194

4 Singer G G, Abbas A K. The Fas antigen is involved in peripheral but not thymic deletion of T-lymphocytes in T- cell receptor transgenic mice. Immunity, 1994, 1: 365 371

5 张国荣, 肖士云, 张平夏, 等. 小鼠胸腺基质细胞单克隆抗体 PF18-3 的特性研究. 中华微生物学和免疫学杂志, 1995, 15(4) : 264 268

6 王 宏, 董海东, 庞学文, 等. 小鼠胸腺基质细胞单抗 PF18-3 的调亡抑制作用及其 识别分子的特征分析. 北京医科大学 学报, 1997, 29(4): 311 313

7 Poccia F, Piselli P, Vendetti S, et al. Heat-shock protein expression on the membrane of T cells undergoing apoptosis. Immunology, 1996, 88: 6 12

8 Martin S J, Reutelingsperger C P M, Mcgahon A J, et al. Early redistribution of plasma membrane phophatidylserine is a general feature of apoptosis regardless of the initiating stimulus: inhibition by overexpression of Bct2 and Abl. J Exp Med, 1995, 182: 1545 1556 
9 Bodmer J L, Burns K, Schneider P, et al. TRAMP, a novel apoptosis-mediating receptor with sequence homology to tumor necrosis factor receptor 1 and Fas (Apo 1/ CD95). Immunity, 1997, 6( 1): 79 88

10 Pan G H, O' Rourke K, Chinnaiyan A M, et al. The receptor for the cytotoxic ligand TRAIL. Science, 1997, 276( 4) : 111 113

\section{痛稳素( nocistatin) 在疼痛调制中的作用}

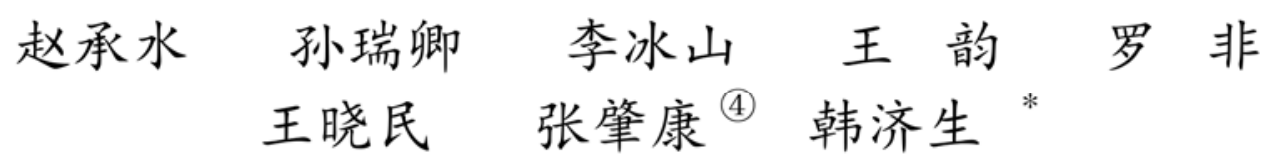

( 北京医科大学神经科学研究所, 北京 100083; (4Phoenix Pharmaceutical Inc., Mountain View, CA, USA.* 联系人)

摘要采用甩尾潜伏期作为伤害感受的指标, 用 von Frey 纤维测量机械性痛觉超敏 (allodynia), 观察给大鼠脑室注射(i. c. v. ) 痛稳素( nocistatin) 能否逆转孤啡肽( orphanin FQ) 的抗 吗啡镇痛作用, 以及脑室注射或脊髓蛛网膜下腔注射( i. t. ) 痛稳素能否影响神经源性痛大鼠 的机械性痛觉超敏. 结果表明, 脑室注射痛稳素不影响大鼠的甩尾潜优期, 也不影响吗啡镇 痛, 脑室注射或脊髓蛛网膜下腔注射痛稳素也不影响神经损伤侧后肢的缩足阈值. 然而, 脑室 注射痛稳素能显著逆转孤啡肽的抗吗啡镇痛作用。由此得出痛稳素在脑水平能逆转孤啡肽的 抗吗啡镇痛作用, 而在脑和脊髓水平均不抑制神经源性痛的机械性痛觉超敏.

\section{关键词 吗啡 孤啡肽 痛稳素 痛觉过敏 痛觉超敏 神经源性痛}

外周神经损伤常产生神经源性痛, 表现为痛觉过敏(痛刺激引起的异常增强的痛反应)、痛 觉超敏( 非痛刺激引起的痛反应) 和自发性疼痛(无明显刺激而产生的痛反应)。目前神经源性 痛的机制不清, 也缺乏有效的治疗. 有报道说, 给小鼠脊髓蛛网膜下腔注射孤儿阿片受体( orphan opioid receptor-like receptor) ORL 1 的内源性激动剂孤啡肽, 能引起痛觉过敏和痛觉超 敏 ${ }^{[1,2]}$, 因而称其为痛敏素( nociceptin). 但 Mogil 及其同事 ${ }^{[3,4]}$ 证明, 给小鼠脑室注射孤啡肽并 不引起痛觉过敏和痛觉超敏, 但能拮抗全身应用吗啡的镇痛作用, 也能拮抗阿片介导的应激性 镇痛及 $\mu, \kappa$ 和 $\delta$ 阿片受体介导的镇痛, 因而是一种抗阿片物质. 我们发现, 给大鼠脑室注射 孤啡肽确能拮抗全身应用吗啡的镇痛作用, 也能拮抗阿片介导的电针镇痛 ${ }^{[5]}$. 最近有人报道, 孤啡肽前体中含有另外一种生物活性肽痛稳素 ${ }^{[6]}$, 在小鼠它能抑制脊髓蛛网膜下腔注射孤啡 肽引起的痛觉过敏和痛觉超敏 ${ }^{[6,7]}$. 本研究的目的是在结扎了 L5 和 L6 脊神经所致的神经源 性痛大鼠身上观察, 痛稳素本身是否有抑制痛敏的作用? 抑或它只是对抗了孤啡肽的抗阿片 作用.

\section{1 材料与方法}

( ) 材料. 实验动物为体重 200 $250 \mathrm{~g}$ 的雌性 SD 大鼠. 孤啡肽和痛稳素为美国 Phoenix Pharmaceutical Inc 产品. 盐酸吗啡为中国青海制药厂产品. 药物均溶于无菌生理盐水 (NS) 中. 吗啡浓度为 $2 \mathrm{mg} / \mathrm{mL}$, 孤啡肽浓度为 $1.6 \mu \mathrm{g} / \mu \mathrm{L}$, 痛稳素浓度由其注药剂量及注药体 